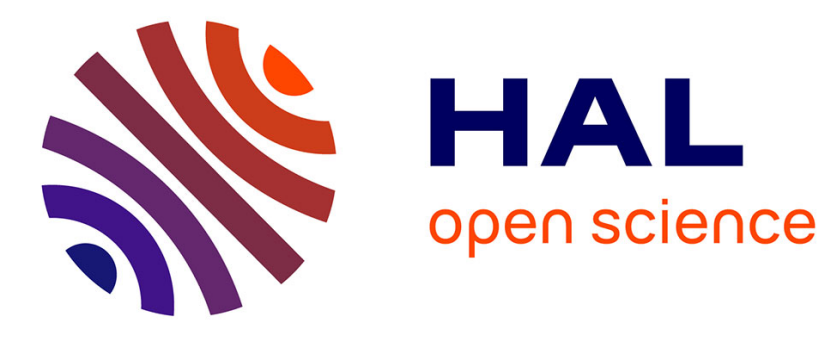

\title{
Médialités biographiques de la pratique scientifique
}

Sonia Dheur

\section{To cite this version:}

Sonia Dheur. Médialités biographiques de la pratique scientifique. Le sujet dans la Cité - Revue internationale de recherche biographique, 2020, Médialités biographiques, pratiques de soi et du monde. Sous la direction de Christine Delory-Momberger et Jean-Claude Bourguignon, Actuels (9/1), p. 233243. 10.3917/lsdlc.hs09.0233 . halshs-02433829

\section{HAL Id: halshs-02433829 \\ https://shs.hal.science/halshs-02433829}

Submitted on 11 Jan 2021

HAL is a multi-disciplinary open access archive for the deposit and dissemination of scientific research documents, whether they are published or not. The documents may come from teaching and research institutions in France or abroad, or from public or private research centers.
L'archive ouverte pluridisciplinaire HAL, est destinée au dépôt et à la diffusion de documents scientifiques de niveau recherche, publiés ou non, émanant des établissements d'enseignement et de recherche français ou étrangers, des laboratoires publics ou privés. 


\section{MÉDIALITÉS BIOGRAPHIQUES DE LA PRATIQUE SCIENTIFIQUE}

\section{Sonia Dheur}

L'Harmattan | « Le sujet dans la cité »

2020/1 Actuels n 9 | pages 233 à 243

ISSN 2112-7689

ISBN 9782343204796

Article disponible en ligne à l'adresse :

https://www.cairn.info/revue-le-sujet-dans-la-cite-2020-1-page-233.htm

Distribution électronique Cairn.info pour L'Harmattan.

(C) L'Harmattan. Tous droits réservés pour tous pays.

La reproduction ou représentation de cet article, notamment par photocopie, n'est autorisée que dans les limites des conditions générales d'utilisation du site ou, le cas échéant, des conditions générales de la licence souscrite par votre établissement. Toute autre reproduction ou représentation, en tout ou partie, sous quelque forme et de quelque manière que ce soit, est interdite sauf accord préalable et écrit de l'éditeur, en dehors des cas prévus par la législation en vigueur en France. Il est précisé que son stockage dans une base de données est également interdit. 


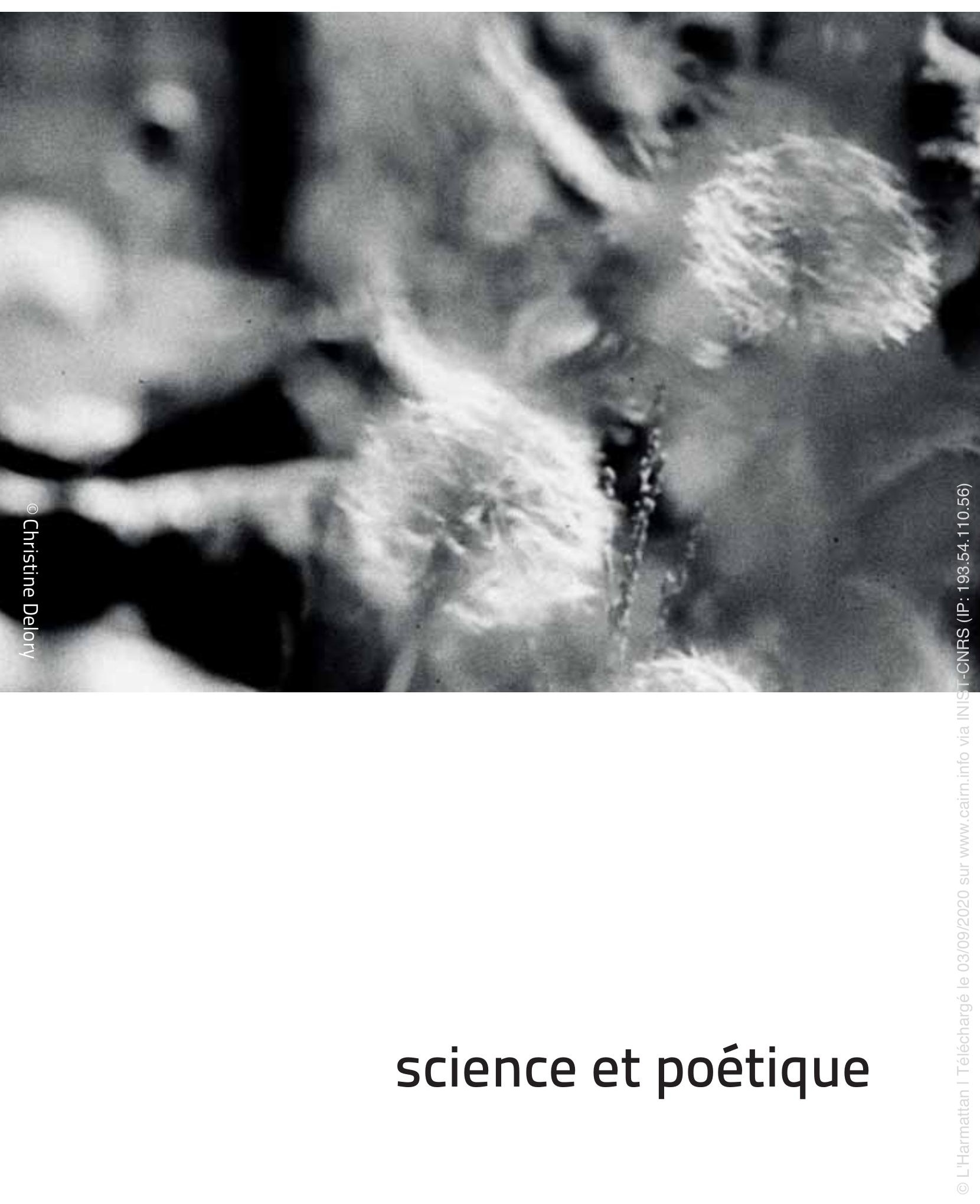




\section{médialités biographiques de la pratique scientifique}

\section{Sonia Dheur ${ }^{1}$}

\section{Résumé}

Après avoir mené une longue activité de recherche fondamentale en biologie, je me suis interrogée sur le processus de biographisation du sujet-cherchant dans et par la pratique scientifique. Avec des collègues chercheurs en sciences humaines et sociales, géographes pour la plupart, mais aussi anthropologues, philosophes, chercheurs en sciences de l'éducation et en littérature, nous nous sommes réunis régulièrement pendant trois ans dans le cadre d'un séminaire visant à explorer la dimension poétique de la pratique scientifique dans le contexte d'élaboration des savoirs académiques qui est le nôtre. Ma communication tente de revisiter cette animation scientifique au prisme de la recherche biographique.

\section{Abstract}

\section{Biographic mediality in scientific research practices}

After having conducted for a long period fundamental research in biology, I started wondering about the biographization process of the epistemic subject (the researcher) in and through the practice of scientific research. With colleagues from social sciences, geography, philosophy, education sciences and literature, we regularly met during a three years period in the context of a seminar dedicated to the exploration of poetic dimensions in science practice, in the contemporary academic environment. My communication attempts to revisit this collective scientific activity in the light of biographic research.

MOTS CLÉS : pratique scientifique, sujet épistémique, réflexivité, récit autobiographique, poétique.

KEYWORDS: science practice, epistemic subject, reflexivity, autobiographic narrative, poetics.

\footnotetext{
1. Sonia Dheur est biologiste et chercheuse au CNRS (INSHS).
} 
La recherche biographique se présente comme une " science du singulier " visant un "savoir de l'un ", un "savoir de l'individu saisi dans sa singularite ${ }^{2}$ ". J'aimerais partager avec vous aujourd'hui une réflexion que je mène sur le « $\mathrm{du}$ » d'une science du singulier et sur le caractère unitaire de l'objet de ce savoir. Cette " « science " de la parole singulière " (Delory-Momberger, 2017a, p. 18) tend vers un savoir « du » sujet en tant que «tourné vers » le sujet, même si on admet la dimension partagée de la recherche biographique, dans laquelle sont engagés ensemble le chercheur et la personne non pas sur mais avec laquelle celui-ci enquête (Delory-Momberger, 2017b). Le savoir auquel je m’intéresse est aussi un savoir « du » sujet, mais entendu comme " œuvre de », " qui s'origine dans » le sujet acteur de la science, le chercheur. $\mathrm{Si}$, comme tout individu, le sujet-cherchant est engagé dans un mouvement d'élaboration de son existence - son propre « mouvement d'éducation " (Delory-Momberger, 2017a, p. 21) -, dans quelle mesure le savoir scientifique produit procède-t-il de son propre travail biographique ? À l'inverse, la pratique scientifique constitue-t-elle pour le chercheur un médium dans son processus de biographisation? Mon expérience m'amène à penser qu'une attention portée sur l'unité du sujet-cherchant - ce qui lui donne sa cohérence - autant que sur sa singularité permet de dépasser la question de l'origine et de la finalité d'un savoir $d u$ sujet.

Pour instruire mon propos, j'ai choisi une mise en abyme. En effet, je commencerai par raconter en quelques mots ce qui m’a amenée à me trouver - à me biographier - ici en ce moment avec vous. Tout de suite me vient l'idée de ce que cette modalité autobiographique représente dans le monde académique de la recherche scientifique (en dehors peut-être de la recherche biographique) : une impudeur, une immodestie, une complaisance, un égotisme déplacés. Je dirais plutôt qu'en bonne enfant d'une société de la deuxième modernité, où l'individu acteur de sa propre vie se doit de faire un travail biographique plus intense pour rétablir une continuité et une cohérence entre les figures et les histoires plurielles de lui-même, c'est ainsi que je choisis de "faire société " (Delory-Momberger, 2017a, p. 14) ici et maintenant. Une autre idée m’interpelle, sous la forme d'une question qui restera ici ouverte : quels savoirs savants, scientifiques, académiques, un chercheur plus ou moins névrosé, pour qui l'injonction " chercher " peut mettre en mouvement une appréhension du monde plus ou moins complexe et difficile, peut-il produire ? Dans quelle mesure la névrose, plus ou moins ordinaire, du chercheur est-elle un problème, un obstacle ou, au contraire, une ressource pour sa communauté et pour le savoir en général ?

Je suis chercheuse au CNRS, en biologie, la " science de la vie, l'étude des êtres vivants", nous dit le Trésor de la langue française, une discipline scientifique à l'origine d'un savoir dont j'attendais toute jeune qu'il m'éclaire sur la vie, donc sur ma vie, et sur sa

\footnotetext{
2. http://lesujetdanslacite.com/1/upload/colloquerbe2019pra_sentationfr_oct2019.pdf
} 
complexité. Beaucoup plus tard, il y a six ans, alors que je menais des recherches dans le champ de la génétique moléculaire depuis près de 20 ans, je me suis demandé pourquoi j'avais toujours travaillé, de près ou de loin, sur l'expression des gènes, mon compagnon sur la reconnaissance du soi et du non-soi, mon chef d'équipe sur la cohésion des chromosomes au cours des divisions cellulaires, etc. Au-delà des mots " expression ", "gènes ", "reconnaissance ", " soi/non-soi ", " cohésion ", " division ", ces faits me semblaient très chargés du point de vue sémiotique et ni simplement fortuits ni contingents ni anodins... C'est d'abord à la psychanalyse qu'il m’a semblé devoir avoir recours pour dénouer ce nœud sémantique. Puis, ignorant tout ou presque des savoirs produits par les sciences humaines et sociales, j'ai commencé par m'initier à l'anthropologie sociale, ce qui m’a permis d'intégrer en 2014 l'UMR Passages à Bordeaux. Il s'agit d'une grosse unité de recherche pluridisciplinaire (comptant environ 230 membres), composée de géographes, d'architectes, de sociologues, etc. qui tous globalement s'intéressent à l'espace et à ses représentations, depuis le corps jusqu’à l'espace numérique en passant par les territoires, l'espace domestique, les parcs nationaux, les migrations humaines. Dans cet environnement, je coordonne depuis 2016 un séminaire sur la thématique des Présentations poétiques du monde.

Cette animation scientifique au sein du laboratoire propose une pause réflexive, un temps de réflexion sur les sujets de nos recherches scientifiques, les chercheurs, nousmêmes, dans les rapports que nous entretenons avec nos objets de recherche et dans les façons que nous avons de mener nos travaux tout au long de leur processus (depuis le choix des thématiques, des terrains, des corpus, en passant par l'expérience ethnographique, la collecte des données, leur analyse, traduction, interprétation, mise en ordre, sens, image, jusqu'à l'écriture, la présentation et la communication d'un discours scientifique). Ainsi, pendant trois ans, seize collègues, presque tous membres de l'UMR Passages, se sont exprimés sur cette question.

Avec le recul, je m’aperçois qu'en proposant cette animation scientifique, j'ai étendu les préoccupations qui étaient à l'origine les miennes propres - personnelles, intimes - à une communauté de chercheurs, d'acteurs de la recherche scientifique. Car ce que je visais, ce qui m'intéressait au fond et à quoi j'incitais autant que possible mes collègues, c'était un travail d'intégration des savoirs qu'ils produisent et des modalités de leur pratique scientifique dans un espace étendu, ouvert à l'environnement social, familial, culturel, idéologique, et dans une temporalité longue, allant de l'enfance, du vécu, des affects aux désirs, aux utopies. Du passé au futur. En passant par ce présent de la présentation, où se loge, me semble-t-il, la dimension poétique, à l'œuvre chaque fois que nous, scientifiques, nous nous exprimons publiquement.

Cette dimension poétique de la parole, j'y avais été sensibilisée au cours de travaux menés précédemment sur la lecture à voix haute, la lecture publique (Dheur, 2017). Je me suis aperçue que la pratique scientifique partageait de nombreux points communs 
avec cette activité culturelle instituée. L'une des choses qui me frappait était l'analogie que je voyais dans le rapport à soi d'une chose extérieure presque sacrée (le texte d'un auteur admiré ou un savoir scientifique) qui, dans le même temps, était incarnée, vécue, vivante pour le lecteur ou le chercheur, et mise à distance, rendue aussi neutre, impersonnelle, sans affect, sans désir, que possible.

Car, pour que les auditeurs trouvent un sens à la lecture à haute voix, il faut que le texte passe au travers du lecteur, par un chemin intérieur, sans toutefois que celuici n'exprime de véritable émotion. Pour y parvenir, le lecteur fait un travail en amont de la lecture publique. Il consiste, dans un premier temps, à ranimer des expériences personnelles passées, incorporées, sensibles et en lien avec le texte lu. Ensuite, par une mise en images de ces expériences, il neutralise cette émotion première pour mieux interpeller son auditoire et lui transmettre du sens à travers le texte qu'il a ainsi fait sien (l'auteur n'est jamais cité par le lecteur lui-même). La proposition que le lecteur fait au public est une présentation poétique du monde, où poétique est à comprendre dans une dimension créative, de façonnement, contenant la continuité, la complexité à la fois de son monde et du contexte de la lecture, du rapport lecteur-texte-public-lieu. De façon similaire, il me semblait que le sujet-cherchant livre un discours à son auditoire ou à son lectorat sur un objet choisi et appréhendé à partir d'une formation, de lectures, d'un cadre académique, d'un terrain, et à travers une histoire et un vécu personnel, sensible, tout ceci impliquant toute sa personne, imprégnée d'expériences de vie et de connaissances, c'est-à-dire une personne à la fois singulière, $u_{n} e^{3}$ et ordinaire.

L'approche narrative autobiographique me semblait être une méthode appropriée pour faire ressortir la dimension poétique du savoir scientifique. Je pensais qu'elle permettrait d'amener des éléments de compréhension du processus de production de ce savoir, depuis l'intérieur, et absents - au moins en apparence - des savoirs tels qu'ils sont institués. Ces derniers ne sont-ils pas le résultat de traductions successives, depuis les carnets de terrain, la correspondance, les croquis, les premières versions de manuscrits jusqu'aux textes publiés, aux manuels de cours et présentations tous publics (Pestre, 2006) ? Je suggérais donc chaque fois de procéder par une narration autobiographique quand j'invitais les intervenants à participer au séminaire.

Au moment où je proposai cette activité collective, en janvier 2016, je savais que la subjectivité de l'ethnologue, à travers le processus réflexif qu'il engage (cette réflexion se prenant elle-même pour objet, cette pensée sur la pensée), ne remettait aucunement en question la légitimité scientifique de celui-ci et était au contraire revendiquée dans la production des savoirs anthropologiques : "L'ethnographe est un savant qui puise

\footnotetext{
3. Dit autrement, " où règne l'unité, dont les parties sont liées entre elles de manière à former un tout " (Dictionnaire de l'Académie française, huitième édition, 1932-1935).
} 
dans son expérience propre les ressources de son savoir. " (Debaene, 2010, p. 74) Cette réflexivité en anthropologie a toujours légitimé les approches poétiques dans la discipline, à travers l'usage de la photographie, du cinéma ou de la littérature ${ }^{4}$. En revanche, je ne connaissais rien ou presque de la longue tradition autobiographique en sciences sociales. Ainsi, petit à petit, je découvrais par exemple les Essais d'ego-histoire coordonnés par Pierre Nora (1987), dans lesquels sept historiens se font les historiens d'eux-mêmes à travers un récit autobiographique visant à mettre en lumière des liens entre expériences personnelles et choix du métier d'historien ; le séminaire " Histoire de vie et choix théoriques " de Vincent de Gaulejac (2014) qui, depuis 25 ans, à travers les itinéraires de grandes figures de la sociologie, de l'anthropologie et de la philosophie, cherche à comprendre comment se construit une pensée, pourquoi et comment elle évolue en fonction de ses racines familiales et affectives, du poids des événements sociaux ou politiques et des rencontres théoriques; les Histoires de géographes où Chantal Blanc-Pamard (1991) importe le projet de Nora auprès de huit collègues africanistes; les Egogéographies où Jacques Lévy raconte son itinéraire intellectuel dans une perspective programmatique d'" objectivation du subjectif " (Lévy, 1995, p. 16), de légitimation du sujet-cherchant; et l'égo-géographie de Yann Calbérac et Anne Volvey (2014a) qui propose d'articuler le biographique et le réflexif au profit d'une approche plus radicale, de nature épistémologique. Il s'agit ici d'interroger la place du géographe dans la construction des savoirs qu'il produit, c'est-à-dire le « procès du sujet " (dans sa dimension subjective et identitaire, consciente et inconsciente) dans «sa " géographie (Calbérac et Volvey, 2014a, p. 10). Cette égo-géographie se distingue du travail rétrospectif d'un individu qui reviendrait sur son parcours intellectuel. Et la méthode utilisée dépasse l'interprétation d'un récit autobiographique, elle fait appel à des matériaux intimes (carnets de terrain, de voyage, de croquis) et à une pratique du terrain avec observations et entretiens "pour étudier le biographique en ses spatialités comme n’importe quel autre objet géographique " (Calbérac et Volvey, 2014a, p. 26). Au-delà de la reconnaissance d'une subjectivité en science, le programme de l'égo-géographie vise l'intégration du sujet épistémique dans les savoirs produits sur les spatialités. Un essai de Calbérac et Volvey (2014b) sur La pensée paysagère d'Augustin Berque instruit remarquablement cette démarche égo-géographique. Les auteurs y dévoilent un processus de construction réciproque du sujet-cherchant et de l'objet scientifique.

\footnotetext{
4. En anthropologie, les arts visuels et la littérature participent de la production des connaissances scientifiques depuis près d'un siècle : initialement à travers les ethnofictions de Robert Flaherty, puis celles de Margaret Mead ou de Jean Rouch, par exemple, et du " deuxième livre de l'ethnographe ", cet ouvrage littéraire venant en sus de la monographie académique, tel L'Afrique fantôme de Michel Leiris ou Tristes tropiques de Claude Lévi-Strauss (Vincent Debaene, 2010, p. 14).
}

5. Le trait d'union souligne pour Calbérac et Volvey l'articulation de l'égo et de la géographie dans ses dimensions épistémologiques. 
Enfin, j'ai découvert la recherche biographique au printemps 2017 à travers un appel à contribution de la revue Le Sujet dans la Cité sur la lecture. Je me suis alors rendu compte que l'autobiographie en sciences sociales était bien plus qu'une méthode, elle est un objet de recherche en soi. Car le récit "n'est pas seulement le produit d'un "acte de raconter", il agit, il a un pouvoir d'effectuation et de transformation." (Delory-Momberger, 2017a, p. 19). Dans le récit biographique, le sujet accomplit un travail de mise en forme et en sens de ses expériences, qui est performatif, qui agit sur ce qu'il raconte et sur celui qui raconte, c'est un puissant moteur de biographisation en tant que travail de construction réciproque de soi et du monde social. Ce qui intéresse le chercheur en recherche biographique, c'est " ce que fait le récit ", " comment le récit à la fois produit et donne à voir la construction singulière " du sujet qui intègre et s'approprie les éléments collectifs du contexte social et culturel (Delory-Momberger, 2017a, p. 19). Ne fallait-il pas, pour saisir le poétique en science, appliquer le mode d'investigation de la recherche biographique, " un soin et une écoute de l'autre attentifs (attentionnés)»(Delory-Momberger, 2017a, p. 20), une attention portée sur l'activité langagière, la parole que le sujet tient sur lui-même, les modalités selon lesquelles il biographie ses expériences?

Il faut comprendre que ces découvertes du biographique en sciences sociales ont été tardives dans le procès du séminaire. Dans l'entre-deux, entre la " naïveté " phénoménologique, qui se déprend par l'épochè de tout savoir constitué, et une vision plus éclairée du biographique en sciences, quelle forme le séminaire avait-il prise, comment les séances se sont-elles déroulées ? Entre janvier 2016 et décembre 2018, treize séances ont eu lieu autour de seize intervenants, majoritairement des chercheurs en géographie sociale, mais aussi en anthropologie, en philosophie, en sciences de l'éducation et en littérature. Chaque séance de trois heures se déroulait en trois parties, une introduction avec présentation de l'intervenant, une communication et une discussion. Des trois termes présentation-poétique-monde, je constate que nos premières rencontres se sont très vite focalisées sur le terme "poétique ». Les premiers locuteurs ont cherché, sans que cela leur soit explicitement demandé, à déterminer en quoi leur pratique ou leurs objets scientifiques pouvaient être qualifiés de "poétiques » : dans la mise en mouvement de l'autre au cours de la transmission du mouvement dansé (comme des mathématiques), dans la relation de tout habitant au monde vue à travers la phénoménologie et l'interactionnisme symbolique, dans le rôle intégrateur du geste comme continuum entre temporalité et spatialité, dans ce que je peux dire de moi, de mon monde, à partir de ce que les sociétés disent d'elles-mêmes, dans ce qui n'est pas entendu ou qui ne parle pas dans ce qui est dit, dans la faculté de percevoir la présence de l'autre, dans ce qui se donne dans un écart, dans l'engagement du chercheur-artiste, dans la déconstruction de nos catégories, etc. Ensuite, progressivement au fil des séances, les intervenants se sont mis à simplement parler de leurs travaux en cours, avec réflexivité, en évoquant certaines difficultés liées au terrain, aux rapports avec 
les informateurs, ou encore des difficultés à faire reconnaître des virages méthodologiques du point de vue épistémologique. Hormis par de rares réponses à mes questions intrusives, les collègues ne se sont pas prêtés à l'exercice de la narration autobiographique tel que je l'avais pensé et essayé de le susciter.

Que se passait-il alors pendant la séance ? Je commençais chaque séance par le compte rendu d'une lecture qui me paraissait intéressante pour penser le poétique et/ou par un retour, à partir de nos discussions, sur la séance précédente sous la forme d'une relecture de celle-ci au prisme du poétique. Par exemple, en introduction de la séance consacrée à Christine Chivallon ${ }^{6}$, j'ai commencé par évoquer un parallèle entre la pensée de «l'entre " de François Jullien, philosophe helléniste et sinologue, et le processus poétique dans la pratique scientifique, en pointant la continuité comme caractère du monde poétique. Lors de la séance suivante, Marina Duféal ${ }^{7}$ nous a parlé de « la face cachée des couleurs ». J'ai introduit son propos en ces termes :

Les couleurs correspondent à l'introduction par l'homme d'une discontinuité dans sa conception, sa perception et sa communication du monde. Mais pour aborder ce fait dans un séminaire qui sáppelle Présentations poétiques du monde, il convient de réintroduire de la continuité dans l'analyse du monde des couleurs. Et pour nous rapprocher au plus près du processus poétique, je vous propose, en introduction à la présentation de Marina, de suivre un fil conducteur, un fil tisseur, qui tente de remettre - ou plutôt de suggérer - du lien, du liant, des fils, là où la modernité, pour comprendre le monde, les a fragilisés et quelquefois brisés. Sans tout expliciter, en conservant de la complexité, je vais me permettre de faire ce qui pourrait vous apparaitre comme une série de digressions, mais qui, de mon point de vue, n'en sont pas. J'espère que vous arriverez à suivre le fil que je déroule - vous ponvez tout aussi bien y dérouler le vôtre d'ailleurs - mais, dans tous les cas, que vous ne vous ennuierez pas.

De cette façon, je suis revenue longuement, en procédant par associations d'idées, à la manière de la psychanalyse, sur la séance précédente consacrée à Christine, où la question de la couleur de la peau avait fini par dominer la discussion. Puis, avant de céder enfin la parole à Marina, j’ai exposé une revue de la littérature sur les liens mis en évidence par certains chercheurs entre le langage, la perception et les représentations des couleurs dans différentes communautés humaines à travers le monde.

\footnotetext{
6. Christine Chivallon est géographe et anthropologue, directrice de recherche au CNRS et membre de l'UMR Passages. Elle est une spécialiste des sociétés à fondement esclavagiste des Amériques, et plus particulièrement de l'espace caribéen. Elle s'est beaucoup intéressée aux relations entre cultures, mémoires et matérialités. Dans le cadre du séminaire Présentations poétiques du monde, elle est intervenue le 23 octobre 2017 avec une présentation qu'elle a intitulée " Usages artistiques et pratiques de pouvoir ».
}

${ }^{7}$ Marina Duféal est géographe, membre de l'UMR Passages et maître de conférences à l'université Bordeaux-Montaigne Elle est intervenue le 26 mars 2018 dans le cadre de notre séminaire. 
Ce tissage, ces associations d'idées tendent à s'approcher davantage du réel en réintroduisant une complexité, qui est la mienne quand je prends la parole, mais qui est co-constituée à partir d'éléments d'une discussion entre collègues, agrémentée d'idées extérieures qui y sont convoquées. Il s'agit d'une forme de biographisation, un processus qui, par le médium de ce séminaire et dans la matérialité d'un ici-et-maintenant, "fait société " (Delory-Momberger, 2017a, p. 14), me permet et permet à mes collègues de nous inscrire dans un groupe humain (dans le contexte du travail), lui-même comme chacun d'entre nous en prise avec un monde social.

La résistance de mes collègues face à l'exercice de la narration autobiographique a finalement permis de révéler que la narration de soi était justement tout ce que le poétique n'est pas. Pour le dire autrement, le poétique émerge justement de l'évitement de la narration de soi. À l'inverse du récit de vie, c'est dans la forme que prend la résistance de l'unité du sujet-cherchant que réside le poétique, dans la constitution de soi en même temps que dans son effacement, dans l'unité de la connaissance savante et de la connaissance ordinaire en soi-même. C'est dans la forme unique et unitaire du sujet (lisant ou cherchant) qui prend la parole en un lieu et un instant donnés que se loge le poétique. Le présent de la présentation du monde par le sujet est le poétique luimême, en tant qu'unité spatio-temporelle de mise en forme socialisée, médiatisée par des systèmes symboliques, qui convient, qui sied aux autres, et qui permet au sujet de s'asseoir, de siéger, d'occuper un siège en société, en s'inscrivant, en s'intégrant dans un contexte socio-historique. Dans le même temps, le présent, dont on aurait tendance à penser qu'il suppose une identité du sujet à un moment donné, est toujours pris dans une dynamique temporelle entre un vécu et une projection, des affects et des désirs, qui supposent toujours une altérité chez le sujet qui ne peut jamais véritablement s'asseoir sur une identité figée (Laplantine, 2000). Le poétique se lit dans la résistance de l'un, de l'unité du sujet et non de son identité, constamment pris entre la constitution de sa singularité en même temps que son effacement, son individuation en même temps que sa socialisation.

Au-delà de leur enjeu épistémologique, les travaux menés dans le séminaire Présentations poétiques $d u$ monde ont une perspective éthique. Anne Volvey, artiste plasticienne et géographe, a mis en place un dispositif de coaching à l'adresse des acteurs de la science ${ }^{8}$, devant " la multiplication des signes de souffrance au travail, de doute et d'épuisement de la créativité intellectuelle des chercheur.e.s et apprenti.e.s chercheur.e.s, dans cette période très délicate pour le monde académique où les épreuves habituellement associées aux actes de recherche se trouvent augmentées des traits de la violence attachée à la néolibéralisation des conditions de travail, à la raréfaction et à la précarisation des

\footnotetext{
${ }^{8 .}$ http://www.soinsetressources.fr/annevolvey.html
} 
emplois, à l'orientation forcée de l'activité scientifique vers les finalités exclusivement technicistes, opérationnelles et de médiation de l'économie de la connaissance ». Dans ce contexte d'élaboration des savoirs académiques, mettre en lumière, prendre conscience, affirmer, assumer et interroger le poétique en science contribue à l'humanisation de la science et de ses travailleurs.

\section{Références bibliographiques}

Blanc-Pamard, C. (dir.). (1991). Histoires de géographes. Paris : Éditions du CNRS.

Calbérac, Y. \& VolveY, A. (2014a). J'égo-géographie... Géographie et cultures, 89-90, 5-32.

- (2014b). "Chose obscure avant qu’on la dise ». Une lecture égo-géographique de La pensée paysagère d'Augustin Berque. Géographie et cultures, 89-90, 151-197.

Debaene, V. (2010). L'adieu au voyage. L'ethnologie française entre science et littérature. Paris : Gallimard.

Delory-Momberger, C. (2014). De la recherche biographique en éducation. Fondements, méthodes, pratiques. Paris : Téraèdre.

- (2017a). De quel savoir la recherche biographique en éducation est-elle le nom ? Le sujet dans la Cité. Revue internationale de recherche biographique, 6, 9-22.

- (2017b). La recherche biographique ou la construction partagée d'un savoir du singulier. Carnets de recherche sur la formation [en ligne], nov. 2017. https://crf.hypotheses.org/136

Dheur, S. (2017). La vie lue à voix haute : présence-absence d'un corps. Le sujet dans la Cité. Revue internationale de recherche biographique, 8, 70-85.

Gaulejac, V. DE (2014). Entre un individu, son histoire et son œuvre, qui produit l'autre? Genèse du séminaire « histoire de vie et choix théoriques ». In Gaulejac V. de \& Lecomte C. (dir.). Itinéraires de chercheurs (p. 9-17). Paris : L'Harmattan.

Laplantine, F. (2000). Je, nous et les autres. Paris : Le Pommier.

LÉVY, J. (1995). Egogéographies. Matériaux pour une biographie cognitive. Paris : l'Harmattan.

NorA, P. (dir.). (1987). Essais d'ego-histoire. Paris : Gallimard.

Pestre, D. (2006). Introduction aux Science Studies. Paris : La Découverte. 